\title{
Holtsch, Doreen
}

\section{Fachdidaktische Kompetenz (künftiger) Lehrender im kaufmännischen Bereich}

Faßhauer, Uwe [Hrsg.]; Fürstenau, Bärbel [Hrsg.]; Wuttke, Eveline [Hrsg.]: Grundlagenforschung zum Dualen System und Kompetenzentwicklung in der Lehrerbildung. Opladen ; Berlin ; Farmington Hills, Mich. : Verlag Barbara Budrich 2011, S. 21-34. - (Schriftenreihe der Sektion Berufs- und Wirtschaftspädagogik der Deutschen Gesellschaft für Erziehungswissenschaft (DGfE))

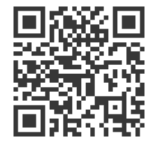

Quellenangabe/ Reference:

Holtsch, Doreen: Fachdidaktische Kompetenz (künftiger) Lehrender im kaufmännischen Bereich - In: Faßhauer, Uwe [Hrsg.]; Fürstenau, Bärbel [Hrsg.]; Wuttke, Eveline [Hrsg.]: Grundlagenforschung zum Dualen System und Kompetenzentwicklung in der Lehrerbildung. Opladen ; Berlin ; Farmington Hills, Mich. : Verlag Barbara Budrich 2011, S. 21-34 - URN: urn:nbn:de:0111-opus-70650 - DOI: 10.25656/01:7065

https://nbn-resolving.org/urn:nbn:de:0111-opus-70650

https://doi.org/10.25656/01:7065

in Kooperation mit / in cooperation with:

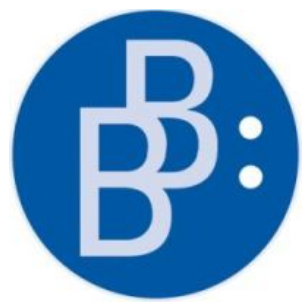

https://www.budrich.de

\section{Nutzungsbedingungen}

Gewährt wird ein nicht exklusives, nicht übertragbares, persönliches und beschränktes Recht auf Nutzung dieses Dokuments. Dieses Dokument ist ausschließlich für den persönlichen, nicht-kommerziellen Gebrauch bestimmt. Die Nutzung stellt keine Übertragung des Eigentumsrechts an diesem Dokument dar und gilt vorbehaltlich der folgenden Einschränkungen: Auf sämtlichen Kopien dieses Dokuments müssen alle Urheberrechtshinweise und sonstigen Hinweise auf gesetzlichen Schutz beibehalten werden. Sie dürfen dieses Dokument nicht in irgendeiner Weise abändern, noch dürfen Sie dieses Dokument für öffentliche oder kommerzielle Zwecke vervielfältigen, öffentlich ausstellen, aufführen, vertreiben oder anderweitig nutzen.

Mit der Verwendung dieses Dokuments erkennen Sie die Nutzungsbedingungen an.

\section{Terms of use}

We grant a non-exclusive, non-transferable, individual and limited right to using this document.

This document is solely intended for your personal, non-commercial use. Use of this document does not include any transfer of property rights and it is conditional to the following limitations: All of the copies of this documents must retain all copyright information and other information regarding legal protection. You are not allowed to alter this document in any way, to copy it for public or commercial purposes, to exhibit the document in public, to perform, distribute or otherwise use the document in public.

By using this particular document, you accept the above-stated conditions of use.

\section{Kontakt / Contact:}

\section{peDOCS}

DIPF | Leibniz-Institut für Bildungsforschung und Bildungsinformation

Informationszentrum (IZ) Bildung

E-Mail: pedocs@dipf.de

Internet: www.pedocs.de

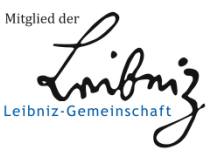


Grundlagenforschung zum Dualen System und Kompetenzentwicklung in der Lehrerbildung 


\section{Uwe Faßhauer}

Bärbel Fürstenau

Eveline Wuttke (Hrsg.)

\section{Grundlagenforschung zum Dualen System und Kompetenzentwicklung in der Lehrerbildung}

Verlag Barbara Budrich Opladen • Berlin • Farmington Hills, MI 2011 
Bibliografische Information der Deutschen Nationalbibliothek

Die Deutsche Nationalbibliothek verzeichnet diese Publikation in der Deutschen

Nationalbibliografie; detaillierte bibliografische Daten sind im Internet über

http://dnb.d-nb.de abrufbar.

(C) Dieses Werk ist im Verlag Barbara Budrich erschienen und steht unter folgender Creative Commons Lizenz: http://creativecommons.org/licenses/by-nc-nd/3.0/de/ Verbreitung, Speicherung und Vervielfältigung erlaubt, kommerzielle Nutzung und Veränderung nur mit Genehmigung des Verlags Barbara Budrich.

\footnotetext{
SOMERIGHTS RESERVED
}

Dieses Buch steht im OpenAccess Bereich der Verlagsseite zum kostenlosen Download bereit (http://dx.doi.org/10.3224/86649461)

Eine kostenpflichtige Druckversion (Printing on Demand) kann über den Verlag bezogen werden. Die Seitenzahlen in der Druck- und Onlineversion sind identisch.

\section{ISBN 978-3-86649-461-9 \\ DOI $\quad 10.3224 / 86649461$}

Umschlaggestaltung: Umschlaggestaltung: bettina lehfeldt graphic design, Kleinmachnow

Verlag Barbara Budrich, http://www.budrich-verlag.de 
Inhaltsverzeichnis

Vorwort.............................................. 7

\section{Teil I: Kompetenzentwicklung in der Lehrerbildung für berufliche Schulen}

Cindy Grzanna

Die Subjektiven Theorien von Absolventen der

Wirtschaftspädagogik über ihre Berufsidentität - Ergebnisse einer explorativen Studie.

Doreen Holtsch

Fachdidaktische Kompetenz (künftiger) Lehrender im

kaufmännischen Bereich

Mareike Junghanns

Die empirische Evidenz der Handlungsfelder von LehrerInnen in den KMK-Empfehlungen zu den Bildungs- und Fachwissenschaften

Ulrike Weyland/ Eveline Wittmann

Zur Einführung von Praxissemestern: Bestandsaufnahme, Zielsetzungen und Rahmenbedingungen.

Volkmar Herkner/Jörg-Peter Pahl

Berufliche Fachrichtungen - Pragmatik, Probleme und Perspektiven............................................... 


\section{Teil II: Grundlagenforschung zum Dualen System}

Stephan Schumann/ Franz Eberle

Bedeutung und Verwendung schwierigkeitsbestimmender Aufgabenmerkmale für die Erfassung ökonomischer und beruflicher Kompetenzen.............................. 77

Daniel Pittich

Studie zur Überprüfung des Zusammenhangs von Verständnis und Fachkompetenz bei Auszubildenden des Handwerks.

Frank Musekamp

Validierung eines Multiple-Choice-Instruments zur Erfassung von Kompetenzen in der Domäne Kfz-Service \& Mandy Hommel

Aufmerksamkeitsverlauf - Fremdbeobachtung und

Eigeneinschätzung.

Raymond Djaloeis/Martin Frenz/Simon Heinen/

Christopher M. Schlick

Diagnose von Energieberatungskompetenz....

Christian Schmidt

Demografischer Wandel und Entwicklung berufsbildender

Schulen

Karin Wirth

Verknüpfung schulischer und betrieblicher Ausbildungsanteile in konsekutiven Ausbildungsformen.... 


\section{Fachdidaktische Kompetenz (künftiger) Lehrender im kaufmännischen Bereich}

Doreen Holtsch

\section{Einleitung}

In verschiedenen wissenschaftlichen Studien war und ist die professionelle Lehrerkompetenz Forschungsgegenstand (z. B. COACTIV, TEDS-M). Zentraler Grund für die Auseinandersetzung mit der Lehrerkompetenz ist, dass sowohl Zusammenhänge zwischen professionell handelnden Lehrern, Merkmalen der Unterrichtsqualität sowie der Schülerleistung untersucht werden. Obgleich in einigen empirischen Untersuchungen diese Zusammenhänge zwischen Lehrerhandeln und Unterrichtsgestaltung in Ansätzen nachgewiesen werden können (vgl. Baumert \& Kunter, 2006; Lipowsky, 2006; Pauli \& Reusser, 2009), besteht nach wie vor Forschungsbedarf.

Ein fachdidaktisch kompetenter Lehrer begünstigt kognitiv herausfordernden Unterricht (anregende Aufgaben), adaptive Unterstützung der Schüler (Geduld, Umgang mit Fehlern) und kann den Lernfortschritt der Schüler voraussagen (Neuweg, 2011, p. 458). Klieme \& Rakoczy (2008, p. 229) stellen dazu fest, dass die kognitive Aktivierung allerdings von Fach zu Fach und je nach Bildungsstufe konkretisiert werden muss. Zur kognitiven Aktivierung gehören aus Sicht von Klieme \& Rakoczy (2008, p. 228) unter anderem herausfordernde, offene Aufgaben im Unterricht.

Eine Reihe von Sondierungsinterviews mit Berliner Lehrkräften im Rahmen der Vorbereitungen auf dieses hier vorzustellende Projekt bestätigt, dass Lehrende Aufgaben für den Unterricht nach eigenen (subjektiven) Kategorien auswählen, verändern und einsetzen oder verwerfen. Lehrende entwickeln darüber hinaus in Ermangelung von passenden Vorlagen häufig eigene Aufgaben, die gleichzeitig anspruchsvoll und motivierend wirken sollen. Allerdings wurden die dabei verwendeten fachdidaktischen Kategorien für den kaufmännischen Unterricht bisher selten in den Forschungsfokus genommen. Deshalb wird im Folgenden ein geplantes Projekt beschrieben, in dem das Aufgabenwissen bei der Auswahl und Veränderung der Aufgaben sowie das aufgabenrelevante Verhalten beim Aufgabeneinsatz bei (künftigen) Lehrenden an kaufmännischen beruflichen Schulen erhoben werden. Im Projekt zur Aufgabenkompetenz kann davon ausgegangen werden, dass sich sowohl das Wissen über Aufgaben als auch das Aufgabenhandeln in konkreten Unterrichtssituationen als Aufgabenkompetenz der Lehrenden abbilden lassen. 
Im vorliegenden Beitrag werden zunächst ausgewählte Befunde der Kompetenzmessung in der Lehrerbildung vorgestellt, die bei der Erhebung der Aufgabenkompetenz zu berücksichtigen sind. Der Beitrag fokussiert alsdann konkrete Forschungsfragen und das geplante Forschungsdesign.

\section{Forschungsstand zu ausgewählten Lehrerkompetenzen}

Mit der Kompetenzdiskussion sind inzwischen verschiedene Vorstellungen über Begriff, Struktur und Niveau verbunden (Hartig \& Klieme, 2006). In der Lehrerbildung lässt sich dennoch Konsens hinsichtlich der Definition herstellen. Breite Verwendung findet die kognitionspsychologische Definition von Kompetenzen nach Weinert. Hier werden unter Kompetenz ,die bei Individuen verfügbaren oder durch sie erlernbaren kognitiven Fähigkeiten und Fertigkeiten, um bestimmte Probleme zu lösen, sowie die damit verbundenen motivationalen, volitionalen und sozialen Bereitschaften und Fähigkeiten, um die Problemlösungen in variablen Situationen erfolgreich und verantwortungsvoll nutzen zu können“"verstanden (Weinert, 2001, p. 27f.).

In Anlehnung an Shulman entwickeln Baumert \& Kunter (2006, p. 482) das Modell professioneller Handlungskompetenz. Sie zeigen, in welche Bereiche und Facetten das Professionswissen strukturiert werden kann und von welchen Aspekten (z. B. Überzeugungen, Motivationen) die professionelle Handlungskompetenz determiniert wird (Abbildung 1).

Baumert \& Kunter (2006, p. 481) betonen, dass sowohl Wissen als auch Können der Kern professioneller Handlungskompetenz von Lehrenden sind. Dabei gelten deklaratives, prozedurales und strategisches Wissen als zentrale Komponenten. Weitere psychologische Kompetenzauffassungen, wie Kompetenz als Fähigkeit zum situationsangemessenen Verhalten und Kompetenz als Expertise stellt Schaper (2009, pp. 170-172) zur Diskussion. Als gemeinsame Schnittmenge der verschiedenen Kompetenzdefinitionen zeichnet sich $\mathrm{ab}$, dass sowohl die Wissens- als auch die Handlungsdimension von Bedeutung sind und sich Kompetenz auf konkrete Domänen (z. B. Wirtschaftswissenschaften) bzw. Situationen (z. B. Aufgabeneinsatz im Unterricht) bezieht. 


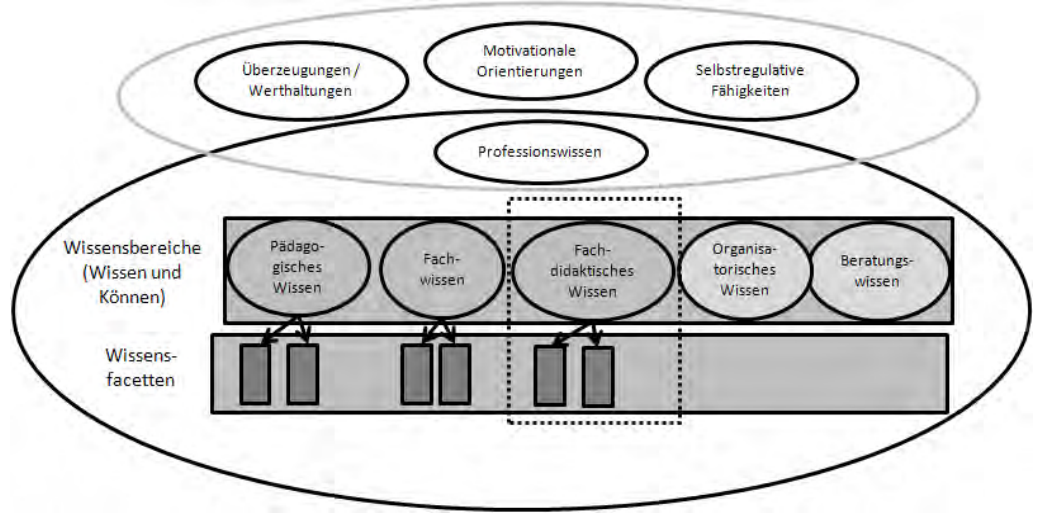

Quelle: Baumert \& Kunter (2006, p. 482) [Modifikationen D. H.]

Bisher realisierte Kompetenzmessungen bei Lehrenden orientierten sich am Modell von Baumert und Kunter (2006) und wurden im allgemeinbildenden Bereich im Unterrichtsfach Mathematik durchgeführt (z. B. COACTIV, MT21, TEDS-M). Die Untersuchungen zeigen, dass sich Fach- und fachdidaktisches Wissen theoretisch wie auch empirisch unterscheiden, auch wenn die Zusammenhangsstärke z. B. im internationalen Vergleich variiert (Blömeke, Kaiser, Döhrmann, \& Lehmann, 2010, p. 237). Es besteht weitgehend Einigkeit darüber, dass Fachwissen eine wichtige Voraussetzung für fachdidaktisches Wissen ist. Je strukturierter und differenzierter das Fachwissen des Lehrenden ist, desto größer ist i.d.R. auch seine fachdidaktische Handlungskompetenz, da fachliche Details unterschiedlich und dennoch richtig erklärt werden können (vgl. Bromme, 1997, p. 194). In den folgenden Betrachtungen wird der Schwerpunkt auf den in Abbildung 1 umrandeten fachdidaktischen Wissensbereich gelegt.

Die fachdidaktische Kompetenz lässt sich auf Basis verschiedener Forschungsansätze (z. B. COACTIV und TEDS-M) in fachdidaktische Facetten strukturieren (Abbildung 2).

In COACTIV wird fachdidaktisches mathematisches Wissen nach dem didaktischen Dreieck in die Facetten Wissen über Aufgaben, Schülervorstellungen sowie Repräsentieren/Erklären unterschieden (Krauss et al., 2008, pp. 234-236). Krauss et al. (2008, p. 250) zeigen beispielsweise, dass das fachdidaktische Wissen bei Gymnasiallehrkräften tendenziell höher ausgeprägt ist als bei Nicht-Gymnasiallehrkäften. Zudem erweist sich fachdidaktisches Wissen als eine zentrale Determinante für das Schülerlernen. 


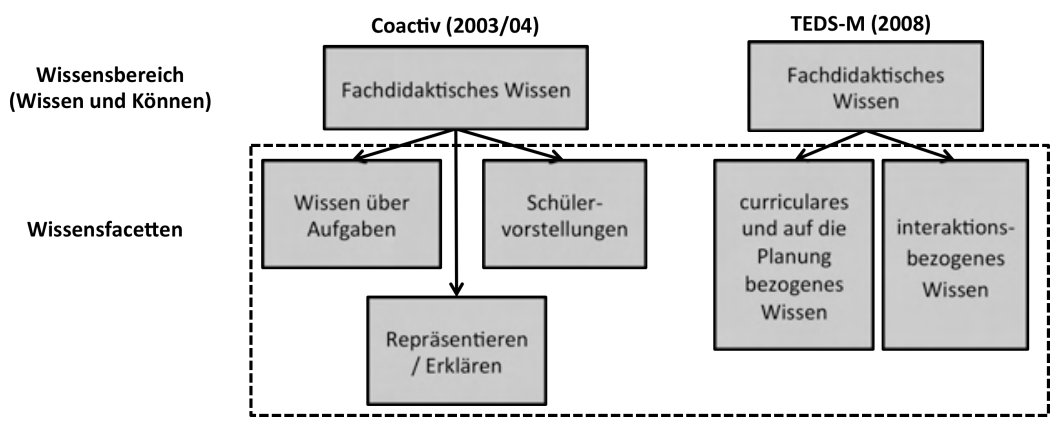

Quelle: Eigene Darstellung in Anlehnung an COACTIV und TEDS-M

In TEDS-M (Döhrmann, Kaiser, \& Blömeke, 2010, p. 175) werden ebenfalls für das Fach Mathematik unterrichtsplanerische und interaktionsbezogene Facetten erfasst. Die Befunde über das stärkere fachdidaktische Wissen bei Lehrenden über die 10. Klasse hinaus werden bestätigt.

Eine weitere Studie zur Kompetenz von Lehrenden im Fachbereich Naturwissenschaften wird von Beck et al. (2008) vorgelegt, in der adaptive Planungs- und Lehrkompetenz unterschieden und mittels Video- und Vignettentest bei jungen und erfahrenen Lehrpersonen in der Schweiz erhoben wurden. In den Ergebnissen verdeutlicht sich der Zusammenhang zwischen Planungs- und Handlungskompetenz. Darüber hinaus ist die adaptive Lehrkompetenz ein Indikator zur Erklärung von Unterschieden in Lernergebnissen.

Die COACTIV-Forschergruppe analysierte ergänzend mathematische Einstiegs-, Klassenarbeits- und Hausaufgaben für die 9. und 10. Jahrgangsstufe. „Aufgaben sind ein Substrat der im Unterricht geschaffenen Lerngelegenheiten und somit ein wichtiges Zeugnis für das kognitive Aktivierungspotential des Unterrichts" (Jordan et al., 2008, p. 86). Das dabei verwendete Aufgabenklassifikationsschema umfasst die vier Dimensionen „Inhaltlicher Rahmen“, „Kognitiver Rahmen“, „Kognitive Elemente des Modellierungskreislaufs“" und „Lösungsraum“ zur Beurteilung von Mathematikaufgaben (Jordan, et al., 2008, pp. 87-91). Im Ergebnis zeigte sich, dass das Aktivierungspotential der Aufgaben niedrig und die Aufgaben selbst sehr homogen ausgeprägt sind (Jordan, et al., 2008, p. 103), was wiederum eine ungünstige Voraussetzung für das Schülerlernen ist. Diese Befunde für das Fach Mathematik werfen die Frage nach dem Aufgabeneinsatz im kaufmännischen Unterricht auf. Sie verdeutlichen gleichzeitig die Notwendigkeit zur Erforschung der Aufgabenkompetenz von Lehrenden.

In der Wirtschaftspädagogik wurden bisher vor allem Untersuchungen zur Gestaltung von Lernumgebungen, z. B. zum Selbstorganisierten Lernen (Sembill, 2004), durchgeführt. Sembill (2008) zeigte insbesondere die positi- 
ven Zusammenhänge zwischen selbstorganisiertem bzw. handlungsorientiertem Lernen und Schülerleistungen bzw. -merkmalen auf.

Die aktuelle Forschung mit dem Fokus auf die professionelle Kompetenz von Wirtschaftspädagogen richtet sich primär auf fachbezogene Überzeugungen (vgl. Seifried, 2009; Seifried, 2010) sowie auf ausgewählte Kompetenzfacetten, z. B. die professionelle Fehlerkompetenz (Seifried \& Wuttke, im Druck). Meist beziehen sich diese Untersuchungen auf eine einzelne wirtschaftswissenschaftliche Subdomäne (bspw. Rechnungswesen).

Eine zentrale Determinante für unterrichtliches Handeln scheint die fachbezogene Sichtweise zu sein. Obwohl die empirische Befundlage zu den Zusammenhängen zwischen Sichtweisen und dem unterrichtlichen Handeln im allgemeinbildenden Bereich äußerst heterogen zu sein scheint (Baumert \& Kunter, 2006, pp. 498-501; vgl. Leuchter, 2009), spricht Seifrieds (2009) Studie für die Berücksichtigung der Überzeugungen und subjektiven Theorien. Er analysierte im Rahmen seiner Habilitation die Sicht von Handelslehrern auf beruflichen Unterricht und identifizierte aufgrund der Sichtweisen drei unterschiedliche Lehrertypen. Die instruktional und konstruktivistisch orientierten Lehrertypen unterscheiden sich bezüglich ihres unterrichtlichen Handelns (z. B. Unterrichtskommunikation, Einsatz Frontalunterricht). Dieser Befund bedeutet für das hier geplante Projekt, dass fachbezogene Überzeugungen unterrichtliches Handeln und damit den Aufgabeneinsatz im kaufmännischen Unterricht beeinflussen können. Deshalb muss die Sichtweise neben anderen Lehrermerkmalen (z. B. sozio-demographische Merkmale) in diesem Forschungsprojekt explizit berücksichtigt werden.

\section{Konzeption der fachdidaktischen Facette „Aufgabenkompetenz ${ }^{66}$}

In Anknüpfung an die im zweiten Abschnitt vorgestellten Definitionen zur Kompetenz wird in diesem Forschungsprojekt davon ausgegangen, dass die Aufgabenkompetenz aus den Dimensionen Wissen und Können besteht. Während sich das Wissen eher in der Unterrichtsplanung zeigt, äußert sich das Können vor allem in der Unterrichtsdurchführung (Tabelle 1). 
Tabelle 1: Dimensionen der Aufgabenkompetenz von Lehrenden

Wissen

Können

Aufgabenwissen
(deklarativ, strategisch), aufgabenbezogenes Können

(prozedural),

im Unterricht,

d. h. Bewertung, Auswahl und Begründung von vorgegebenen Aufgaben bei der Unterrichtsplanung

d. h. Umsetzung der Aufgaben

Kontext

Erfassung der Vorbereitung und der Umsetzung von vorgegebenen Aufgaben im Wirtschaftslehreunterricht in ausgewählten kaufmännischen Berufen

Quelle: Eigene Darstellung

Ausgehend von den hier vorgestellten Befunden werden die fachdidaktischen Kompetenzstrukturen von COACTIV und TEDS-M (in Abbildung 2 umrandet) auf das Konzept der Aufgabenkompetenz übertragen. Sie wird sowohl unter einer unterrichtsplanerischen (Aufgabenwissen) als auch unter einer interaktionsbezogenen Perspektive (aufgabenbezogenes Können) betrachtet. Das Aufgabenwissen des Lehrenden umfasst die Fähigkeit, problemorientierte Aufgaben nach fachdidaktischen Ansätzen zu konstruieren, auszuwählen und zu modifizieren. Die Aufgabe im Unterricht (unmiss)verständlich einzusetzen, gehört zur Dimension des Aufgabenkönnens. Während die erste Dimension eher auf die deklarativen und strategischen Wissenskomponenten abzielt, wird die prozedurale Komponente durch die zweite Dimension repräsentiert. Beide Dimensionen bilden die Aufgabenkompetenz der Lehrenden $a b$, die als eine fachdidaktische Kompetenz in der Domäne Wirtschaftswissenschaften zu verstehen ist.

Der Begriff der Fachdidaktik ist jedoch nicht immer klar abgegrenzt, was sich sehr deutlich in der Domäne Wirtschaftswissenschaften zeigt (siehe z. B. Achtenhagen, 1984, p. 222; Euler \& Hahn, 2007, p. 76; Jongebloed \& Twardy, 1983; Kaiser \& Kaminski, 1999, pp. 13-14). Bei allen Unterschieden wird gleichfalls deutlich, dass für die Aufgabeneinschätzung (resp. Erhebung des Aufgabenwissens) und den Aufgabeneinsatz domänenspezifische, und hier vor allem inhaltliche und methodische Kriterien zum Tragen kommen.

Das Wissen über Aufgaben wird aus Gründen der Vergleichbarkeit mit Hilfe eines Kategorienschemas erhoben, anhand dessen (künftige) Lehrende identische Aufgaben bezüglich fachdidaktischer Kategorien einschätzen müssen. Die in COACTIV verwendeten Kategorien gelten für das Fach Mathematik und können nur begrenzt auf den wirtschaftlichen Kontext angewendet werden. Obwohl einige der Kategorien als erste Anhaltspunkte für die Einschätzung von Aufgaben gelten, werden fachdidaktische Kategorien für die Domäne Wirtschaftswissenschaften entwickelt. Tabelle 2 illustriert 
eine Auswahl verschiedener inhaltlicher und methodischer Kategorien für den kaufmännischen Unterricht.

Tabelle 2: Kategorien zur Einschätzung von Aufgaben (Auswahl)

\begin{tabular}{|l|l|}
\hline \multirow{4}{*}{$\begin{array}{l}\text { Die Wirtschaftssituation der Aufgabe } \\
\text { bezieht sich auf folgenden Bereich }\end{array}$} & Situation als Staats- und Wirtschaftsbürger \\
\cline { 2 - 2 } & Situation am Arbeitsplatz als Sachbearbeiter/in \\
\cline { 2 - 2 } Verstehen von Fachbegriffen & Situation des privaten Konsumenten \\
\hline \multirow{5}{*}{ Wirtschaftliche Konzepte } & keine Fachbegriffe \\
\cline { 2 - 2 } & aus einem Gebiet (z. B. BWL oder VWL) \\
\cline { 2 - 2 } & aus mehreren Gebieten gemischt \\
\hline & wirtschaftliche Konzepte wiedergeben \\
\cline { 2 - 2 } & wirtschaftliche Konzepte anwenden \\
\cline { 2 - 2 } & wirtschaftliche Konzepte verknüpfen \\
\cline { 2 - 2 } & wirtschaftliche Konzepte infrage stellen \\
\hline
\end{tabular}

Im Unterricht werden verschiedene Aufgabentypen eingesetzt, z. B. Lernaufgaben, Übungsaufgaben und Testaufgaben. Neben Fragen gelten Aufgaben als kleinste methodische Einheit. Diese können nach Euler \& Hahn (2007, p. 352) als Problem oder als Übung/Routine formuliert werden. Im geplanten Projekt wird der Schwerpunkt ausschließlich auf Lern- und Übungsaufgaben gelegt. Von der Lernsituation als im Lernfeldkonzept kleinste geschlossene curricular-methodische Einheit wird bewusst abgewichen, weil sich diese über eine größere Zahl von Unterrichtseinheiten erstreckt (siehe zur Umsetzung von Lernfeldern z. B. Kremer \& Sloane, 1999a, 1999b). Ausuntersuchungsökonomischen Gründen wird die Aufgabe als kleinere Einheit in den Blick genommen. Dies ermöglicht, dass Aufgaben unabhängig davon analysiert werden können, in welcher Form und wie konsequent das Lernfeldkonzept umgesetzt wird.

Zusammengefasst wird als „Aufgabe“ für die Untersuchung der Aufgabenkompetenz von Lehrenden im kaufmännischen Unterricht eine nach fachdidaktischen Ansätzen entwickelte problemhaltige Lern- oder Übungsaufgabe verstanden, die im Unterricht in einer vorgegebenen Zeit in ausgewählten kaufmännischen Ausbildungsberufen bearbeitet wird. 


\section{Forschungsfragen}

Zur fachdidaktischen Kompetenzfacette „Aufgabenkompetenz“ im kaufmännischen Unterricht ergeben sich zusammengefasst verschiedene Forschungsfragen ${ }^{1}$, die untersucht werden sollen:

1. Welche nach fachdidaktischen Ansätzen entwickelten Aufgaben repräsentieren das kaufmännische Handlungsfeld?

2. Wie ist die Aufgabenkompetenz (Aufgabenwissen und aufgabenbezogenes Können) von (künftigen) Lehrenden ausgeprägt?

3. Welche Zusammenhänge bestehen zwischen Aufgabenwissen und aufgabenrelevantem Können (Verhalten)?

4. Wie unterscheidet sich die Aufgabenkompetenz zwischen ReferenzExperten und (künftigen) Lehrenden?

5. Welche Determinanten beeinflussen die Aufgabenkompetenz?

\section{Geplantes Forschungsdesign zur Untersuchung der Aufgabenkompetenz}

In diesem Projekt soll die Aufgabenkompetenz von (künftigen) Lehrenden an kaufmännischen Schulen im quasilängsschnittlichen Design untersucht werden. Zielgruppe des Projektes sind deshalb Master-Studierende der Wirtschaftspädagogik, Lehrende im Vorbereitungsdienst sowie Lehrende an kaufmännischen Schulen oder an Wirtschaftsgymnasien.

Das in Modulform geplante Projekt bereitet im ersten Modul „Vorarbeiten“ die Untersuchung vor (Tabelle 3). In diesem Modul werden Aufgaben entwickelt, die das kaufmännische Handlungsfeld repräsentieren (Forschungsfrage 1). Diese Aufgaben und das Kategorienschema zur Einschätzung der Aufgaben werden Referenz-Experten vorgelegt. Tabelle 3 gibt einen Überblick über die geplanten Teilschritte.

1 Die Forschungsfragen sind chronologisch nach den im Folgenden beschriebenen Module aufgestellt. 
Tabelle 3: Modul 1, „Vorarbeiten“

Modul 1, „Vorarbeiten“

\begin{tabular}{ccl}
$\begin{array}{c}\text { Forschungsziele / } \\
\text { - fragen }\end{array}$ & - & $\begin{array}{l}\text { Sichtung und Entwicklung von repräsentativen Aufgaben für das } \\
\text { kaufmännische Handlungsfeld }\end{array}$ \\
Stichprobe & $\bullet$ & $\begin{array}{l}\text { Referenz-Experten } \\
\text { (z. B. Fachdidaktiker, Praktiker, Fachleiter, Schulinspektoren) }\end{array}$ \\
Methodisches Vorgehen & $-\quad \begin{array}{l}\text { Delphi-Untersuchung zur Bestimmung und Validierung der Auf- } \\
\text { gabensammlung und -bewertung }\end{array}$ \\
\hline
\end{tabular}

Quelle: Eigene Darstellung

Im ersten Schritt werden Aufgaben recherchiert, die im kaufmännischen Unterricht eingesetzt werden. Neben unterschiedlichen fachdidaktischen Zugängen sind auch weitere Aufgabenmerkmale wie Zahl der Lösungswege $\mathrm{zu}$ berücksichtigen. Die von den Verlagen zur Verfügung gestellten Unterrichtsmaterialien und IHK-Prüfungen quantitativ stark besetzter kaufmännischer Ausbildungsberufe (z. B. Industriekaufmann/-frau, Kaufmann/frau im Einzelhandel) stellen die Recherchebasis dar. Die Rechercheergebnisse flieBen in einer Synopse zusammen, in der die gemeinsame inhaltliche Schnittmenge von Aufgaben über verschiedene Ausbildungsberufe abgeleitet werden kann.

Gleichzeitig wird das Kategoriensystem entwickelt, nach dem die Aufgaben eingeschätzt werden müssen. Erstes Ziel dieses Moduls ist also die Entwicklung eines hinreichend großen Aufgabenpools, der in mehreren Ausbildungsberufen eingesetzt werden kann, sowie eines fachdidaktisch orientierten Kategoriensystems, mit dem die Aufgaben bewertet werden können.

Die konstruierten Aufgaben werden anschließend in einer DelphiUntersuchung von Referenz-Experten gesichtet und reflektiert. Als ReferenzExperten sollen Akteure aus der Lehreraus- und Weiterbildung gewonnen werden. Zudem sollen Praktiker beteiligt werden, die das kaufmännische Handlungsfeld kennen. Dies bedeutet, dass u. a. Fachdidaktiker, Fachwissenschaftler, Praktiker, Fachleiter aus der zweiten Phase der Lehrerausbildung und Schulinspektoren in der Expertengruppe vertreten sind. Die DelphiUntersuchung dient zur Validierung der im ersten Modulteil gesammelten Aufgaben. Zweites Ziel des Moduls ist die Bewertung der Aufgaben durch die Experten nach dem entwickelten Kategoriensystem.

Um im zweiten Modul Aufschluss über das Aufgabenwissen (Forschungsfrage 2) zu erlangen, werden die vorbereiteten Aufgaben (künftigen) Lehrenden vorgelegt (Tabelle 4). Für einen Quasi-Längsschnitt werden dabei drei Zielgruppen beteiligt: Master-Studierende, die im Praktikum erste Unterrichtserfahrungen sammeln, Lehrende im Vorbereitungsdienst sowie Lehrende, die auf eine mindestens fünfjährige Berufserfahrung nach dem Vorberei- 
tungsdienst zurückblicken können. Es wird vermutet, dass die Lehrenden mit der längsten Unterrichtserfahrung über das differenzierteste Aufgabenwissen verfügen und am stärksten mit dem Expertenrating übereinstimmen.

Tabelle 4: Modul 2 „Aufgabenwissen“

Modul 2 ,Aufgabenwissen“

$\begin{array}{cl}\begin{array}{c}\text { Forschungsziele / } \\ \text { - fragen }\end{array} & \text { - Ausprägung des Aufgabenwissens } \\ & \text { - Unterscheidung des Aufgabenwissens zwischen Zielgruppen } \\ \text { Stichprobe } & \text { - Studierende im letzten Praktikum }(\mathrm{N}=20) \\ & \text { - Lehrende im Vorbereitungsdienst }(\mathrm{N}=50) \\ & \text { - Lehrende }(\mathrm{N}=50 ;>5 \text { Jahre }) \\ \text { Methodisches Vorgehen } & \text { - Aufgabenbewertung } \\ & \text { - Entscheidungen Aufgabeneinsatz } \\ & \text { - Lehrerfragebogen }\end{array}$

Quelle: Eigene Darstellung

Die Lehrenden bewerten die Aufgaben anhand des Kategorienschemas und entscheiden, ob und aus welchen Gründen sie die vorliegende Aufgabe (nicht) einsetzen würden. Darüber hinaus geben die Lehrenden an, welche konkreten Lösungswege in ihrer Klasse wahrscheinlich gewählt werden, wie viele Schüler die Aufgabe richtig lösen etc. Diese Angaben werden von Kontextvariablen ergänzt, z. B. dem Nutzungsverhalten von Medien (genutzte und eingesetzte Lehrbücher). Ferner werden weitere Determinanten der Aufgabenkompetenz, wie sozio-demographische Angaben, Sichtweisen und Motivationen der Lehrenden erhoben.

Im dritten Modul setzen Lehrende in einer Berufsschulklasse in den ausgewählten Ausbildungsberufen eine der Aufgaben ein, um ihr aufgabenrelevantes Verhalten (ebenfalls Forschungsfrage 2) erheben zu können (Tabelle $5)$. 
Tabelle 5: Modul 3 „Aufgabenrelevantes Können“

Modul 3 „Aufgabenrelevantes Können“

\begin{tabular}{|c|c|}
\hline $\begin{array}{l}\text { Forschungsziele / } \\
\text { - fragen }\end{array}$ & $\begin{array}{l}\text { - aufgabenbezogenes Können (Unterrichtsmuster) } \\
\text { - Unterscheidung des aufgabenrelevanten Verhaltens zwischen Ziel- } \\
\text { gruppen }\end{array}$ \\
\hline Stichprobe & $\begin{array}{l}\text { - Studierende im letzten Praktikum }(\mathrm{N}=20) \\
\text { - Lehrende im Vorbereitungsdienst }(\mathrm{N}=50) \\
\text { - Lehrende }(\mathrm{N}=50 ;>5 \text { Jahre })\end{array}$ \\
\hline Methodisches Vorgehen & $\begin{array}{l}\text { - Videografie - standardisierte und explorative Auswertung } \\
\text { - Lehrerfragebogen }\end{array}$ \\
\hline
\end{tabular}

Quelle: Eigene Darstellung

Aus Gründen der Vergleichbarkeit werden Berufsschulklassen des 2. Ausbildungsjahres gewählt, die bereits mit verschiedenen wirtschaftswissenschaftlichen Unterrichtsinhalten vertraut sind. Die Klasse wird beim Aufgabeneinsatz zufällig in Experimental- und Kontrollgruppe geteilt. Der Lehrende setzt die Aufgabe in der Kontrollgruppe mittels standardisierter Instruktionen ein. In der Experimentalgruppe dürfen die Instruktionen variiert werden. Die Aufgabe selbst soll aus Gründen der Vergleichbarkeit unverändert bleiben. Die Lehrenden werden beim Einsatz der Aufgaben videografiert. Dieses Vorgehen ermöglicht die Dokumentation des aufgabenrelevanten Verhaltens (z. B. Instruktionen, Beantwortung von Schülerfragen, Lösungshinweise) und soll zeigen, welche Kategorien kognitiver Stimuli und Strukturhilfen die Lehrenden wählen und welche aufgabenbezogenen Muster sich identifizieren lassen.

Verschiedene Beobachtungsstudien (z. B. TIMSS und IPN-Videostudie) geben wesentliche Anregungen für Beobachtungskategorien im Unterricht. In TIMSS wurde beispielsweise festgestellt, dass landesspezifische Unterrichtsmuster mit einer gemeinsamen Schnittmenge über die sieben beteiligten Länder hinweg existieren (Givvin, Hiebert, Jacobs, Hollingsworth, \& Gallimore, 2005).

Nach der Videoaufnahme, d. h. nach dem Aufgabeneinsatz im Unterricht, sollen die Lehrenden zum aufgenommenen Unterricht Auskunft geben, inwieweit Unterrichtssituation und Schülerverhalten repräsentativ waren (z. B. Seidel, Prenzel, \& Kobarg, 2005, p. 16f.).

Im vierten Modul wird der Zusammenhang zwischen den zwei Kategorien Aufgabenwissen und aufgabenrelevantem Verhalten analysiert (Forschungsfragen 3 bis 5), damit die in Tabelle 1 vorgestellte Struktur der Aufgabenkompetenz empirisch geprüft werden kann. 
Tabelle 6: Modul 4 „Vertiefende Analysen“

Modul 4 ,Vertiefende Analysen“

\begin{tabular}{cl}
\hline $\begin{array}{c}\text { Forschungsziele / } \\
\text { - fragen }\end{array}$ & - Zusammenhänge zwischen Aufgabenwissen und aufgabenrelevantem \\
& Können \\
& - Determinanten der Aufgabenkompetenz \\
- Zusammenhang zwischen Aufgabenkompetenz und Schülerleistung & \\
Stichprobe & - Schüler einer Klasse (Kontroll-/Experimentalgruppe) \\
Methodisches Vorgehen & - Schülerlösung und Schülerfragebogen \\
\hline
\end{tabular}

Quelle: Eigene Darstellung

In diesem Modul werden die Zusammenhänge zwischen Aufgabenwissen und aufgabenrelevantem Können sowie Determinanten (sozio-biographische Merkmale) erhoben. Hierbei wird der Frage nachgegangen, inwieweit sich (künftige) Lehrenden verschiedener Professionalisierungsetappen in ihrer Aufgabenkompetenz unterscheiden.

Zur Analyse der Zusammenhänge zwischen Aufgabenwissen und aufgabenrelevantem Lehrerverhalten zur Schülerleistung, werden wiederum die Schülerlösungen gesammelt und ausgewertet. Parallel werden einige Kontrollvariablen erhoben, wie z. B. kognitive, motivationale, metakognitive Schülermerkmale. Weiterhin sollen das themenbezogene und das ökonomische Vorwissen der Lernenden berücksichtigt werden.

\section{Zusammenfassung}

Das hier vorgestellte Projekt verfolgt das Ziel, einen externen Referenzrahmen für repräsentative Aufgaben und ihre Bewertung im kaufmännischen Bereich zu entwickeln. Darüber hinaus ermöglicht das Projekt Aussagen zur Aufgabenkompetenz von (künftigen) Lehrenden und ihrer Rückwirkungen auf Unterricht und Schülerleistungen. Das Projekt leistet mit der fachspezifischen Kategorisierung von Aufgaben einen Beitrag zur Weiterentwicklung der Wirtschaftsdidaktik. Im Ergebnis werden Aufschlüsse über die fachdidaktische Kompetenz und strukturelle Zusammenhänge sowie niveaubezogene Ausprägungen erwartet. Die Konsequenzen reichen für Wirtschaftspädagogen von der Gestaltung der Studienprogramme in der ersten und zweiten Phase der Lehrerausbildung bis zu möglichen Impulsen für die Lehrerweiterbildung. 


\section{Literatur}

Achtenhagen, F. (1984). Didaktik des Wirtschaftslehreunterrichts (1 ed.). Opladen: Leske + Budrich.

Baumert, J., \& Kunter, M. (2006). Stichwort: Professionelle Kompetenz von Lehrkräften. Zeitschrift für Erziehungswissenschaft, 9(4), 469-520.

Beck, E., Baer, M., Guldimann, T., Bischoff, S., Brühwiler, C., Müller, P., et al. (2008). Adaptive Lehrkompetenz: Analyse und Struktur, Veränderbarkeit und Wirkung handlungssteuernden Lehrerwissens. Münster [u. a.]: Waxmann.

Blömeke, S., Kaiser, G., Döhrmann, M., \& Lehmann, R. (2010). Mathematisches und mathematikdidaktisches Wissen angehender Sekundarstufen-I-Lehrkräfte im internationalen Vergleich. In S. Blömeke, G. Kaiser \& R. Lehmann (Eds.), TEDS-M. Professionelle Kompetenz und Lerngelegenheiten angehender Mathematiklehrkräfte für die Sekundarstufe I im internationalen Vergleich (pp. 197-238). Münster [u.a.]: Waxmann.

Bromme, R. (1997). Kompetenzen, Funktionen und unterrichtliches Handeln des Lehrers. In F. E. Weinert, N. Birbaumer \& C. F. Graumann (Eds.), Enzyklopädie der Psychologie. Psychologie des Unterrichts und der Schule (pp. 177-212). Göttingen [u.a.]: Hogrefe.

Döhrmann, M., Kaiser, G., \& Blömeke, S. (2010). Messung des mathematischens und mathematikdidaktischen Wissens: Theoretischer Rahmen und Teststruktur. In S. Blömeke, G. Kaiser \& R. Lehmann (Eds.), TEDS-M. Professionelle Kompetenz und Lerngelegenheiten angehender Mathematiklehrkräfte für die Sekundarstufe I im internationalen Vergleich (pp. 169-196). Münster [u.a.]: Waxmann.

Euler, D., \& Hahn, A. (2007). Wirtschaftsdidaktik (2., aktualisierte Aufl. ed.). Bern [u.a.]: Haupt.

Givvin, K. B., Hiebert, J., Jacobs, J. K., Hollingsworth, H., \& Gallimore, R. (2005). Are there national patterns of teaching? Evidence from the TIMSS 1999 video study. [Proceedings Paper]. Comparative Education Review, 49(3), 311-343.

Hartig, J., \& Klieme, E. (2006). Kompetenz und Kompetenzdiagnostik. In K. Schweizer (Ed.), Leistung und Leistungsdiagnostik (pp. 127-143). Heidelberg: Springer Medizin.

Jongebloed, H.-C., \& Twardy, M. (1983). Strukturmodell Fachdidaktik Wirtschaftswissenschaften (SMFW). In M. Twardy (Ed.), Kompendium Fachdidaktik Wirtschaftswissenschaften Band 3/Teil I. Wirtschafts-, Berufs- und Sozialpädagogische Texte (pp. 163-203). Düsseldorf: Verlagsanstalt Handwerk.

Jordan, A., Krauss, S., Löwen, K., Blum, W., Neubrand, M., Brunner, M., et al. (2008). Aufgaben im COACTIV-Projekt: Zeugnisse des kognitiven Aktivierungspotentials im deutschen Mathematikunterricht. Journal für MathematikDidaktik, 29(2), 83-107.

Kaiser, F.-J., \& Kaminski, H. (1999). Methodik des Ökonomie-Unterrichts : Grundlagen eines handlungsorientierten Lernkonzepts ; mit Beispielen (3., vollst. überarb. Aufl. ed.). Bad Heilbrunn: Klinkhardt.

Klieme, E., \& Rakoczy, K. (2008). Empirische Unterrichtsforschung und Fachdidaktik. Outcome-orientierte Messung und Prozessqualität des Unterrichts. Zeitschrift für Pädagogik, 54(2), 222-237. 
Krauss, S., Neubrand, M., Blum, W., Baumert, J., Brunner, M., Kunter, M., et al. (2008). Die Untersuchung professionellen Wissens deutscher MathematikLehrerinnen und -Lehrer im Rahmen der COACTIV-Studie. Journal für Mathematik-Didaktik, 29(3/4), 223-258.

Kremer, H. H., \& Sloane, P. F. E. (1999a). Lernfelder - Motor didaktischer Innovationen? Kölner Zeitschrift für Wirtschaft und Pädagogik, 14(26), 37-60.

Kremer, H. H., \& Sloane, P. F. E. (1999b). Lernfelder implementieren - erst Umsetzungserfahrungen lernfeldstrukturierter Curricula (Vol. 17). München: Ludwig-Maximilians-Universität München.

Leuchter, M. (2009). Die Rolle der Lehrperson bei der Aufgabenbearbeitung : unterrichtsbezogene Kognitionen von Lehrpersonen. Münster [u. a.]: Waxmann.

Lipowsky, F. (2006). Auf den Lehrer kommt es an. Empirische Evidenzen für Zusammenhänge zwischen Lehrerkompetenzen, Lehrerhandeln und dem Lernen der Schüler. Weinheim [u. a.]: Beltz.

Neuweg, G. H. (2011). Das Wissen der Wissensvermittler Problemstellungen, Befunde und Perspektiven der Forschung zum Lehrerwissen. In E. Terhart, H. Bennewitz \& M. Rothland (Eds.), Handbuch der Forschung zum Lehrerberuf (pp. 451-477). Münster [u. a.]: Waxmann.

Pauli, C., \& Reusser, K. (2009). Zum Einfluss von Professionalität auf die Qualität von Lehr-Lern-Prozessen. In O. Zlatkin-Troitschanskaia, K. Beck, D. Sembill, R. Nickolaus \& R. Mulder (Eds.), Lehrprofessionalität. Bedingungen, Genese, Wirkungen und ihre Messung (pp. 679-690). Weinheim [u.a.]: Beltz.

Schaper, N. (2009). Aufgabenfelder und Perspektiven bei der Kompetenzmodellierung und -messung in der Lehrerbildung. In N. Schaper, A. H. Hilligus \& P. Reinhold (Eds.), Kompetenzmodellierung und -messung in der Lehrerbildung. Lehrerbildung auf dem Prüfstand (Vol. 2, pp. 166-199). Landau: VEP.

Seidel, T., Prenzel, M., \& Kobarg, M. (2005). How to run a video study : technical report of the IPN Video Study. Münster [u.a.]: Waxmann.

Seifried, J. (2009). Unterricht aus der Sicht von Handelslehrern. Frankfurt am Main [u.a.]: Lang.

Seifried, J. (2010). Sichtweisen von Lehrkräften an kaufmännischen Schulen. Zeitschrift für Berufs- und Wirtschaftspädagogik, 106(2), 199-219.

Seifried, J., \& Wuttke, E. (im Druck). Diagnose von und Umgang mit Schülerfehlern als Facette der professionellen Kompetenz von Lehrpersonen. In N. Schaper, H. Kremer, A. H. Hilligus \& P. Reinhold (Eds.), Kompetenzmodellierung und messung in der Lehrerbildung. Lehrerbildung auf dem Prüfstand.

Sembill, D. (2004). Prozessanalysen Selbstorganisierten Lernens. Abschlussbericht AZ. Se 573/4-2 an die Deutsche Forschungsgemeinschaft im Rahmen des Schwerpunktprogramms ,Lehr-Lern-Prozesse in der kaufmännischen Erstausbildung“ Available from http://www.uni-bamberg.de/fileadmin/uni/fakultaet en/sowi_lehrstuehle/wirtschaftspaedagogik/Dateien/Forschung/Forschungsprojek te/Prozessanalysen/DFG-Abschlussbericht_sole.pdf

Sembill, D. (2008). Zeitver(sch)wendung in Bildungsprozessen. In M. Gläser-Zikuda \& J. Seifried (Eds.), Lehrerexpertise : Analyse und Bedeutung unterrichtlichen Handelns (pp. 19-46). Münster [u. a.]: Waxmann.

Weinert, F. E. (2001). Leistungsmessungen in Schulen. Weinheim [u.a.]: Beltz. 